\title{
12 \\ Frameworks for Decision-Making in Design for the Aging
}

\author{
Halime Demirkan
}

\section{Introduction}

Different aspects of design epistemology have been emphasized in the design literature for several years. Because of the nature of the design process, designers solve problems that are not well defined and the methods that they use are not fully understood (Demirkan 1998, 2005). Although the final design goals may be unclear at the beginning of the design process, the sub-goals must be well defined by the designer throughout the entire design process (Akin 1986; Cross 2006; Lawson 1990).

As Cross explains, while designers deal with vague problems, their "mode of thinking is constructive" and they use "codes that translate abstract requirements into concrete objects" (Cross 1982). When Cross distinguishes design from other ways of knowing, he emphasizes the importance of knowing, thinking, and acting (Cross 2001). Further, Cross adds that designers develop an intellectual awareness by making explicit analyses and comparisons of the paradigms by layering the approaches of Simon's "rational problem-solving" and Schon's constructivism of "reflective practice" (Cross 2001; Schon 1983, 1987; Simon [1969] 1996).

A "rational problem-solving" approach means that designers are "concerned with how things 'ought' to be... in order to attain goals and to function." According to Simon, "certain phenomena are 'artificial' in a very specific sense; they are as they are only because of a system being molded, by goals or purposes, to the environment in which it lives" (Simon [1969] 1996; Visser 2010).

Constructivism of "reflective practice" problem-solving, as explained by Schon, is an approach that integrates or links thought and action with reflection. It involves 
thinking about and critically analyzing one's actions with the goal of improving one's professional practice. Engaging in reflective practice requires individuals to assume the perspective of an external observer in order to identify the assumptions and feelings underlying their practice and then to speculate about how these assumptions and feelings affect practice (Imel 1992).

In the following essay, the focus is on how designers solve problems in the conceptual design phase; a phase in which information-processing and decision-making are very intensive as a consequence of generating and evaluating alternative ideas. The aim is to demonstrate an epistemological and methodological approach that permits researchers to capture, describe, prioritize, act on, and evaluate alternative design solutions. Thus, the main emphasis of the essay is to provide a framework for understanding how methods and knowledge are linked within the cognitive strategies of design when creating interiors. For example, in design, the designer constructs a conceptual model of the artifact by abstracting knowledge from previous experiences and information stored in the memory. These conceptual representations are linked both with the external forms of knowledge as sketches or drawings and with the internal representations of the model as visual imagery.

The framework for understanding how this works is discussed through a focus on aging adults. This population was selected as the focus because of the complexity of issues that confront designers when creating interior spaces for maturing populations. Within the scope of this discussion, this complexity is addressed only to explain the framework; a complete study would involve many more layers of modeling.

\section{A Framework for the Acquisition and Generation of Knowledge in the Conceptual Phase of Design Problem-Solving}

Although design as a discipline has its specific characteristics, measures, and procedures, these have not been extensively analyzed, described, or formalized. Therefore, it can be stated that there is no theoretical knowledge base for design. Knowledge acquisition is a different process in design and yet there is no formal methodology that has proven effective. Knowledge is generated and accumulated through action. Designing for maturing populations and judging the results is the accepted general model. This is a cyclical process in which knowledge is used to create designs, and designs are evaluated to build knowledge.

As pointed out previously, designers construct a conceptual model of the built environment by abstracting knowledge from previous experiences and information stored in their memory (Demirkan, Pultar, and Ozguc 1992). Knowledge may be the truth or a rule of thumb that usually does not change over time, while the latter two can be modified according to the designer. Some of this knowledge is composed of facts and unlikely to change, while other knowledge is more nebulous and subject to change. 
There are three major knowledge sources from which designers gather information when problem-solving: the relevant community of designers, experts, and clients; the relevant media, such as books, drawings, codes, journals, internet sources, and visual outlets; and the relevant domain accumulated by observing and modeling (Demirkan 1998).

In the acts of building and using design knowledge, questions are asked, answers are given, and decisions are made. The process through which these three factors are formed is the solution to success in building knowledge (Owen 2007). If the question in knowledge using or doing (building) is theoretical or methodological, in other words, if the designer is seeking to understand a phenomenon or process, the design is a conceptual model. The output of the design process is a design solution and not an artifact.

The design model describes how the conceptual model is realized with representational techniques that are the various drawings or computer representations of the artifacts. The conceptual models are more concerned with "finding" or discovering; design models are oriented toward "making" and inventing.

The process of knowledge using and building is fundamentally the same for the conceptual and design models. The differences are more in the purpose of the activity. The purpose of designing is to transform a concept into a design description in such a way that the artifact being described is capable of producing the determined function. Design requires a representational framework that has sufficient expressive power to capture the nature of the concepts that support the design process.

Certain aspects of maturing populations determine the decision-making processes used in the design of interior spaces. These aspects may involve age, sex, anthropometric dimensions, education level, ability level, use of assistive devices, and income level. Such important interacting and variable design elements must be considered in a design problem-solving process to satisfy the needs of maturing populations. Moreover, determining these requirements becomes a difficult process because of the imprecise and uncertain specifications and priorities of maturing populations (Afacan and Demirkan 2010). Thus, the requirements of the interior determine the decisions made in the design process. This being the case, knowledge is not a set of descriptions but an analytical abstraction (Demirkan 2005).

The conceptual design phase has four stages: concrete experience is followed by observations and reflections that lead to the formation of abstract concepts and generalizations, which are then tested through sketches. This is a circular process in which the actions are repeated until an optimum solution is found (Demirbas and Demirkan 2003).

\section{Linking Methods and Knowledge within Cognitive Strategies}

In order to provide an optimal design solution for maturing populations, designers need to operate within an effective cognitive strategy. Over the last four decades, 
design research has largely concentrated on designers' interactions with the design process and their engagement with design problems as a sequence of strategies (Akin 1986; Cross 1989; Lawson 1990; Schon 1983; Simon [1969] 1996). According to the literature, a cognitive strategy is the general plan of a sequence of particular actions employed by a designer throughout the design process (Afacan and Demirkan 2011). In other words, a designer's strategy refers to how a particular designer tackles a design problem.

The implementation of a design strategy varies from one designer to another because design problem-solving is based on the subjective interpretations of each designer (Demirkan 1998). This means that an effective cognitive strategy for one designer may be quite different from the cognitive strategy of another designer (Dorst and Cross 2001).

Even though design strategies may vary from designer to designer, according to Cross there are two primary types of cognitive strategies that designers rely upon: convergent and divergent (Cross 1989). The convergence-based design strategy is concerned with selecting the most appropriate solution from the alternatives regarding the objectives of the design problem; the divergence-based strategy deals with producing a wide range of alternatives (Afacan and Demirkan 2011). Cross suggests that convergent thinkers are successful in selecting the most feasible solution among the alternatives and in satisfying the requirements of the final design phase, while divergent thinkers are good at conceptual design and generate a wide range of alternative solutions (Cross 1989).

It is not possible to rely solely upon a convergence- or divergence-based strategy in the conceptual design phase. Hence, the ideal strategy in the conceptual design phase should be one which supports the dynamic nature of a design that will generate the most satisfactory solution. In this respect, Liu, Bligh, and Chakrabarti stated that it is important to carry out a thought process where as many different solutions as possible are explored for each task, and then, by moving to the convergentthinking portion of the design process, the non-usable ones can be eliminated (Liu et al. 2003).

Liikkanen and Perttula maintain that during the conceptual phase of the design process idea generation involves repeated analysis and synthesis cycles to investigate problem decomposition. The completion of multiple divergent and convergent activities at each level of solution abstraction allows a designer to generate a reasonable number of concepts that are manageable at each level of the solution domain (Liikkanen and Perttula 2009).

According to Gero and McNeill, in a collaborative design process there are three micro-design strategies that designers use. These are: (1) analysis of a solution; (2) proposal of a solution; and (3) reference to explicit strategies (Gero and McNeill 1998). These strategies are related to application knowledge, knowledge and requirements of the design domain, and design strategies. The analysis of the solution refers to the application of knowledge, the proposal of a solution refers to the knowledge and requirements of the design domain, and the implementation of explicit strategies refers to overall design strategies and approaches. 
Gero and McNeill's research showed that the designer was more involved in the micro-design strategy of "proposing a solution" in the conceptual phase, with the designer cycling between the "proposal of a solution" and the "analysis of a solution" when he or she spent almost equal time in both phases (Gero and McNeill 1998).

A research study conducted by Sagun and Demirkan examined Gero and McNeill's three micro-design strategies (Sagun and Demirkan 2009). The results showed that designers usually rejected immediately what were deemed to be unsatisfactory solutions, settling on a single solution instead of providing choices. Further, it was found that the greatest number of critiques focused on the first micro-design strategy proposed by Gero and McNeill (i.e., analysis of a solution).

In another study Demirkan found that the greatest number of critiques focused on the micro-design strategy of analysis (Demirkan 2005). In this same study Demirkan found that "reference to explicit strategies" occurred the second most frequently as opposed to Gero and McNeill's assertion that this micro-design strategy is least frequent. In a later study, Sagun and Demirkan found that the smallest number of critiques were related to explicit design strategies (Sagun and Demirkan 2009).

\section{A Discussion of the Conceptual Phase of Designing for Maturing Populations}

The following discussion is offered in an effort to increase understanding of how designers utilize knowledge and knowledge sources, and what strategies they use in design problem-solving during the conceptual phase. Understanding the human diversity of maturing populations is critical when creating interior environments for older adults. Because of the imprecise and uncertain specifications and priorities of a maturing population, the determination of requirements is a difficult process that challenges designers to seek information from knowledge sources both within and outside the parameters of most design problems (Afacan and Demirkan 2010).

This discussion, however, is not intended to address all the issues associated with the creation of interiors for maturing populations. Rather, it is intended, through the use of this example, to offer insights into how designers approach problem-solving and set priorities, while considering the significant differences that exist among maturing populations.

As a starting point in the design process, exploration of the knowledge domains associated with maturing populations is critical. These domains include information about such things as the personal and physical characteristics of aging adults and the social constructions of maturing populations. The data involve the age, sex, and anthropometric dimensions of the maturing population.

In humans, aging is a multi-dimensional process of physical and social change. The different age periods of the maturing population encompass different changes in physical and psychological characteristics, attitude, and behavior. The sex of the maturing population is important because of the different ways males and females 
use an interior based on cultural differences. Knowledge of human body size alone is not sufficient; information is needed about functional capabilities, mobility, and limitations in performing certain tasks.

By drawing from these knowledge sources, a designer develops a clearer picture of the type of environment best suited to a maturing population demographic. These knowledge domains fall under the general category of Design Knowledge and more specifically under the domains of Human and Interior Descriptions.

\section{The knowledge domain of personal characteristics in maturing populations}

The knowledge domain of Personal Characteristics provides insights for the designer during the conceptual phase of design regarding the idea of functional status. Functional status is a term that reflects a person's ability to handle the basic activities of daily living such as feeding, dressing, ambulating, bathing, transferring from bed to toilet, and grooming, and the ability to communicate. Such afflictions as arthritis, heart disease, diabetes, and restricted vision and hearing are common in maturing people and affect their functional status.

Further examination of the data in the knowledge domain of Personal Characteristics of maturing individuals reveals that people experience degenerative bodily changes at different time intervals. Also, as a maturing population continues to age, people may have problems moving around (mobility deficits), manipulating objects (dexterity deficits), and receiving sensory information (sensory deficits) (Demirbilek and Demirkan 1998).

Maturing populations often experience feelings of isolation because of the death of family members and peers. To help lessen isolation concerns, it is important that interior spaces for maturing populations include areas where people can meet and interact with each other. For example, in structures where the occupants are ambulatory the placement of benches on the staircase landing or in the corridors is a simple way to encourage interaction and interdependence among older adults (Demirkan 2000).

\section{The knowledge domain of status in maturing populations}

Income level and cultural background are two important aspects that determine the perceived status of maturing populations. Through an examination of the Status Domain of income and culture, designers gain an appreciation for the lifestyle, values, and subcultures of maturing populations that can be applied during the conceptual phase of design.

From an early age, shared meanings of the domains of income and culture are learned through social contact with others. As a result, cultural differences are learned factors, rather than genetic (Newell 1997). Thus, two people raised in 
different cultures may have common needs or desires, but different ways of satisfying them. For example, sitting down is a worldwide activity, but whether one sits on the floor or on a chair has major implications for the design of interiors for maturing populations.

\section{The Knowledge Domain of Abilities in Maturing Populations}

The knowledge domain of Abilities in maturing populations falls under the umbrella of universal design and within the context of "design for all." According to Story, Mueller, and Mace, there are five important subcategories that must be taken into consideration in the creation of a successful interior space for maturing adults. These are: (1) cognition, (2) vision, hearing and speech, (3) body function, (4) arm function and hand function, and (5) mobility (Story et al. 1998).

The subcategory of cognition in maturing individuals affects the usability of an interior or a product. Therefore "the variety of abilities in receiving, comprehending, interpreting, remembering, or acting on information" should be considered in the conceptual phase when designing for maturing interior populations (Story et al. 1998b). Moreover, cognition level is dependent upon the age and ability level of the population, as well as on the characteristics of the environment, or of the task conducted. Thus, it is important for designers to incorporate into their problem-solving approach the understanding that maturing populations may include individuals with diminished memory and reasoning skills, as well as individuals who may become easily fatigued or distracted.

The subcategory knowledge domain that deals with vision offers useful insights in the conceptual phase of design in regard to how older adults perceive visual stimuli. For example, older adults may not be able to clearly perceive visual details, focus on nearby and distant objects, discriminate objects from their background, perceive contrasts in color and brightness, and adapt to high and low lighting levels. Additionally, maturing populations may be distracted in a visual environment where stimuli are dense, and other individuals may feel fatigued by excessive visual tasks or may have visual impairments such as presbyopia, glaucoma, or cataracts.

Exploration of the knowledge domain subcategory of hearing and speech provides designers with information about the ability of maturing populations to localize sound. For example, often older adults have difficulty in separating auditory information from background noise, perceiving both high- and low-pitched sounds, or carrying on a conversation (Story et al. 1998). In addition, the level of hearing disability is often age-dependent and the attention of maturing populations may be divided among several auditory sources.

Understanding the functioning level of the body/arm/hand category of older adults informs the decision-making of a designer in the conceptual phase. In the context of "design for all," "body function means consideration of the variety of human abilities in performing common tasks... such as: physical exertion; achiev- 
ing, maintaining and changing posture; maintaining equilibrium and breathing" (Story et al. 1998). Moreover, according to Story et al., the functional level of body/ arm/hand is dependent on age, disability level of the population and the characteristics of the environment or task conducted.

Lastly, the subcategory of mobility is an important knowledge area that informs design decision-making. Central to design conceptualization for maturing populations is an understanding that there are a variety of possible mobility issues, and that even common tasks such as rising from a seated position, standing upright, walking, or kneeling often are challenging for older adults and maturing populations (Story et al. 1998).

\section{The Knowledge Domain of the Built Environment: Physical and Social Environments}

The physical and social environment subcategories of the knowledge domain of the Built Environment are important to consider when conceptualizing interior spaces for maturing populations. These two subcategories offer the designer insights into how to create environments for older adults that provide for the interaction of individuals with other people while still maintaining individual privacy.

It goes without saying that when dealing with the physical environment there are certain codes and regulations that must be considered in designing space for older adults. However, other design factors may be equally important to consider in providing a positive experience for older adults in their built environment. Such things as layout and configuration, functionality, maximum distances, ease of servicing, accessibility, and related technologies also have a bearing and must be part of the design conceptualization process.

As discussed earlier, as a designer moves from the conceptual phase into the design phase of a project, the focus necessarily shifts from theoretical to more pragmatic aspects associated with the physical environment. Primary among these is the selection of appropriate mechanical systems that will enhance and/or support the experience of maturing adults in their physical environment. For example, the specification of electrical systems that address illumination levels, glare control, increased stimulus contrast, and reduction in visual clutter should be a priority.

Other mechanical systems, which are the most supportive of an aging process, should also be selected. These include such things as heating/cooling, ventilation, communication, and security systems. For example, recognition of the fact that the body's ability to regulate its temperature declines with age supports the design decision to place thermostats where individuals with lessened mobility and visual acuity can easily see and control them.

The social environment subcategory of the Built Environment Domain informs the conceptualization process by introducing designers to knowledge sources that address the relationship between the patterns of an activity and the ability of a physical environment to support that activity. Issues like privacy, territoriality, 
personal space, and social interaction permeate these knowledge sources and are critical references when conceptualizing space for maturing populations.

\section{The Prioritization of Requirements}

As can be seen from the previous discussion, there are many variable and interacting design issues to be addressed when creating interior environments for maturing populations. The changing needs and diversity of maturing populations dictate that there will be some level of uncertainty about what is deemed to be a successfully designed interior environment for older adults.

The purpose of this essay is to provide a framework for understanding how methods and knowledge are linked within the cognitive strategies of design when creating interiors for maturing populations. Since all the requirements of such environments cannot be satisfied for each individual's situation, the designer must set priorities by tapping into the knowledge domain of "design for all" factors. Although "design for all" suggests a holistic approach that does not include individuals with disabilities as a specialized group (Demirkan 2007), it should be recognized that the accumulated knowledge of this domain can offer useful information during the conceptual design phase.

The "design for all" literature does not provide concrete answers to the kinds of systematic procedures and methods a designer should use to most effectively identify and set priorities and incorporate user needs into interior spaces for maturing populations (Canadian Human Rights Commission 2006; Center for Universal Design 1997, 2000). It has been suggested that, since all design requirements cannot be equally satisfied for this population, a designer should sift through the knowledge domains and sources available to them and make priority decisions based on the relative importance and order of each requirement (Ozkaya and Akin 2006).

An examination of the term priority shows that a priority is considered to be either the quantity and/or the importance of a requirement (Lehtola, Kauppinen, and Kujala 2004). The literature offers several examples of ways to set priorities that may be helpful to the design community when creating interiors for maturing populations (Karlsson, Wohlin, and Regnell 1998; Karlsson, Thelin, Regnell, Berander, and Wohlin 2007).

One such approach is called the Analytic Hierarch Process (AHP). In this approach to priority-setting, a designer compares two requirements to each other to determine which one is more important, and to what extent. The most obvious drawback to this approach is that it is time-consuming. Since all unique pairs of requirements have to be compared in this approach, the required effort can be substantial. In a design project, time is often of the essence, therefore this approach may make sense only for small-scale projects (Khari and Kumar 2013).

A less rigorous AHP approach is proposed by Wiegers and based on weighted assessments of perceived value, relative penalty, anticipated cost, and technical risks 
(Wiegers 2003). The fundamental difficulty with Wiegers' approach is that the value assigned to a given requirement lacks the specificity necessary to determine whether or not the requirement meets core values.

In contrast to the AHP approach to setting priorities is the Value Oriented Prioritization (VOP) process. When using the VOP approach, a framework is established that identifies core values and the relative relationships among those values (Azar, Smith, and Cordes 2007).

The literature suggests that Quality Function Deployment (QFD) is another effective approach to set design requirement priorities. According to its developer, Dr. Yoji Akao, QFD is a "method to transform user demands into design quality, to deploy the functions forming quality, and to deploy methods for achieving the design quality into subsystems and component parts ... [QFD] is a way to assure design quality while the product is still in the design stage" (Akao 1994). By incorporating the voice of the users, QFD can help designers to transform the needs of users into characteristics that are appropriate - thereby establishing the design requirement priorities.

Lastly, the Planning Game (PG) technique can be used to help establish design priorities. According to the literature, PG is one of the most recognized prioritization techniques in use today (Lehtola and Kauppinen 2006). This approach involves end-users and proposes that priorities can be placed on an ordinal scale so that a ranked order among requirements can be established. In this approach requirements are written down by the end-users and divided into different piles.

Beck suggests that when using the PG technique for priority-setting there should be three piles: those requirements which are essential for functionality; those that are less essential but provide significant value; and those that would be nice to have. At the same time as the end-users sort the cards, the programmer (or in this case the designer) estimates how long each requirement will take to implement and sorts the requirements into three different piles of risk, i.e., the ones that can be estimated precisely, the ones that can be estimated reasonably well, and the ones that cannot be estimated at all. The end result of this sorting is a sorted list of requirements on an ordinal scale that is flexible and can be scaled up or down quickly to establish the order of priorities (Beck 2001).

\section{The Application of Priority-Setting Techniques in Research}

In the design of interior environments for maturing populations it may be necessary to apply variations of each of the priority-setting techniques previously discussed. For example, the PG technique may be chosen because it is user-friendly and quick and easy to implement. On the other hand, the AHP technique may be a more appropriate approach to priority-setting because of its precision, ability to be combined with other techniques, and capacity for creating a systematic formulation of complex requirements that is suitable for handling complex design requirements (Afacan and Demirkan 2010). 
In a research study to explore how a QFD approach worked in the context of design problem-solving for maturing populations, Demirbilek and Demirkan classified all data, such as answers to pre-set questions, proposals, requirements, and ideas, according to the Quality Function Deployment system. The results of the study showed that the use of a QFD approach to priority-setting clearly established the relationships among the elderly users' requirements, their stated wishes, and the technical design specifications (Demirbilec and Demirkan 2004).

In another study, by Demirkan and Olgunturk, the aim was to determine and set priorities for the issues that are important for diverse user groups within the context of "design for all." A survey was conducted with four types of users: individuals with physical disabilities, individuals with visual impairments, the elderly, and fully functioning adults (Demirkan and Olgunturk 2013). The results of the survey were statistically tested to obtain a priority listing of essential items in interior environments designed for all. The following nine "design for all" factors resulted from the study and are listed below in priority order from highest to lowest:

1 Adequate illumination level in all spaces

2 Ease of use in the kitchen

3 Adequate space for approach and use

4 Adequate contrast between essential information and its surroundings

5 Ease of use in accessories

6 Functional vertical circulation

7 Provision of privacy and safety in bathroom

8 Safety of floors in all spaces

9 Accessibility to all spaces

Statistical analysis showed that there was a significant difference among user groups in all factors; however, all the groups did not differ significantly from each other in each factor.

\section{Conclusion}

The goal of this essay was to examine how methods and knowledge are linked within the cognitive strategies of the design process when creating interior environments for maturing populations. As discussed throughout, during the conceptual phase the designer constructs a mental image of the artifact to be produced by abstracting knowledge from previous experiences and information stored in the memory.

As stated by Liikkanen and Perttula, idea generation involves repeated analysis/ synthesis cycles to investigate problem decomposition in the conceptual design phase. So, carrying out multiple divergent and convergent activities at each level of solution abstraction allows a designer to generate a reasonable number of concepts that are manageable at each level of the solution domain (Liikkanen and Perttula 2009). 
The design process as a creative act should be explored within a model framework considering visual imagery and external representation. These conceptual representations are linked both with the external forms of knowledge (sketches) and with the internal representations (imagery) of the model. Sketches as external representations quicken the process, improve results, and aid the restructuring of the problem. This process is especially helpful for designers who have learned to use the sketch as a tool, and encourages the discovery of new information by combining new input and previous knowledge (Bilda and Demirkan 2003; Demirkan 2005; Suwa and Tversky 1997).

The design problem-solving process - consisting of the analysis, synthesis, and evaluation phases - utilizes acquired knowledge to support decision-making through cognitive design strategies. The systematic presentation and manipulation of design requirements is essential, requiring the selection of an appropriate prioritysetting technique to best meet the needs of maturing populations.

\section{References}

Afacan, Y. and Demirkan, H. 2010. "A priority-based approach for satisfying the diverse users' needs, capabilities and expectations: a universal kitchen design case," Journal of Engineering Design 21(2-3): 315-343.

Afacan, Y. and Demirkan, H. 2011. "An ontology-based universal design knowledge support system for the conceptual design phase," Knowledge-Based Systems 24(4): 530-541.

Akao, Y 1994. "Development history of quality function deployment," in QFD: The Customer Driven Approach to Quality Planning and Deployment. Minato, Tokyo: Asian Productivity Organization, p. 339.

Akin, O. 1986. Psychology of Architectural Design. London: Pion.

Azar, J., Smith, R. K., and Cordes, D. 2007. "Value oriented requirements prioritization in a small development organization," IEEE Software 32-73.

Beck, K. 2001. Extreme Programming: Explained (7th ed.). Boston: Addison-Wesley.

Bilda, Z. and Demirkan, H. 2003. "An insight on designers' sketching activities in traditional versus digital media," Design Studies 24(1): 27-50.

Canadian Human Rights Commission. 2006. International Best Practices in Universal Design: A Global Review. CD-ROM. Canada: Betty Dion Enterprises.

Center for Universal Design. 1997. The Principles of Universal Design. Version 2.0. Raleigh: North Carolina State University.

Center for Universal Design. 2000. "Architecture and interior design," in Universal Design Exemplars. CD-ROM. College of Design, Raleigh: North Carolina State University.

Cross, N. 1982. “Designerly ways of knowing," Design Studies 3(4): 221-227.

Cross, N. 1989. Engineering Design Methods. Chichester: John Wiley \& Sons.

Cross, N. 2001. “Designerly ways of knowing: design discipline versus design science," Design Issues 17(3): 49-55.

Cross, N. 2006. Designerly Ways of Knowing. London: Springer.

Demirbas, O. O. and Demirkan, H. 2003. "Focus on architectural design process through learning styles," Design Studies 24(5): 437-456. 
Demirbilek, O. and Demirkan, H. 1998. "Involving the elderly in the design process," Architectural Science Review 41(4): 157-163.

Demirbilek, O. and Demirkan, H. 2004. "Universal product design involving elderly users: a participatory design model," Applied Ergonomics 35(4): 361-370.

Demirkan, H. 1998. "Integration of reasoning systems in architectural modeling activities," Automation in Construction 7(2-3): 229-236.

Demirkan, H. 2000. "A knowledge-based system for the design of elderly residences," in J. G. Mangin and M. Miramond (eds.), Proceedings of 2nd International Conference on Decision Making in Urban and Civil Engineering. Lyon: Université de Valenciennes, pp. 825-836.

Demirkan, H. 2005. "Generating design activities through sketches in multi-agent systems," Automation in Construction 14(6): 699-706.

Demirkan, H. 2007. "Housing for the aging population," European Review of Aging and Physical Activities 4(1): 33-38.

Demirkan, H. and Olgunturk, N. 2013. "A priority-based 'design for all' approach to guide home designers for independent living," Architectural Science Review. Under review.

Demirkan, H., Pultar M., and Ozguc, B. 1992. "A knowledge-based space planning system," Architectural Science Review 35(1): 3-7.

Dorst, K. and Cross, N. 2001. "Creativity in the design process: co-evolution of problemsolution," Design Studies 22(5): 425-437.

Gero, J. S. and Mc Neill, T. 1998. "An approach to the analysis of design protocols," Design Studies 19(2): 1-61.

Imel, S. 1992. Reflective Practice in Adult Education. ERIC Digest No. 122. Columbus, OH: ERIC Clearinghouse on Adult Career and Vocational Education.

Karlsson, L., Thelin, T., Regnell, B., Berander, P., and Wohlin, C. 2007. "Pair-wise comparisons versus planning game partitioning: experiments on requirements prioritization techniques," Empirical Software Engineering 12(1): 3-33.

Karlsson, J., Wohlin, C., and Regnell, B. 1998. "An evaluation of methods for prioritizing software requirements," Information and Software Technology 39(14-15): 939-947.

Khari, M. and Kumar, N. 2013. "Prioritization techniques for software requirements," Journal of Global Research in Computer Science 4(1): 38-43.

Lawson, B. 1990. How Designers Think: The Design Process Demystified. London: Butterworth Architecture.

Lehtola, L. and Kauppinen, M. 2006. "Suitability of requirements prioritization methods for market-driven software product development," Software Process Improvement and Practice 11(1): 7-19.

Lehtola, L., Kauppinen, M., and Kujala, S. 2004. "Requirements prioritization challenges in practice," in Proceedings of 5th International Conference on Product Focused Software Process Improvement. Kansai Science City, Japan, pp. 497-508.

Liikkanen, L. A. and Perttula, M. 2009. "Exploring problem decomposition in conceptual design among novice designers," Design Studies 30(1): 38-59.

Liu, Y. C., Bligh, T., and Chakrabarti, A. A. 2003. “Towards an 'ideal' approach for concept generation," Design Studies 24(4): 341-355.

Newell, P. B. 1997. "A cross-cultural aspects of environmental design," Environment and Behavior 29(4): 495-514.

Owen, C. 2007. "Design thinking: notes on its nature and use," Design Research Quarterly 2(1): 16-27. 
Ozkaya, I. and Akin, O. 2006. "Requirement-driven design: assistance for information traceability in design computing," Design Studies 27(3): 381-398.

Sagun, A. and Demirkan, H. 2009. "On-line critiques in collaborative design studio," International Journal of Technology and Design Education 19(1): 79-99.

Schon, D. A. 1983. The Reflective Practitioner: How Professionals Think in Action. New York: Basic Books.

Schon, D. A. 1987. Educating the Reflective Practitioner: Towards a New Design for Teaching in the Professions. San Francisco: Jossey-Bass.

Simon, H. A. [1969] 1996. The Sciences of the Artificial (3rd ed.). Cambridge, MA: MIT Press.

Story, M. F., Mueller, J. L., and Mace, R. L. 1998. The Universal Design File: Designing for People of All Ages and Abilities (rev. ed.). Raleigh: North Carolina State University, Center for Universal Design.

Suwa, M. and Tversky, B. 1997. "What do architects and students perceive in their design sketches? A protocol analysis," Design Studies 18(4): 385-403.

Visser, Willemien. 2010. "Simon: design as a problem-solving activity, Collection 2 (Art + Design \& Psychology) 11-16.

Wiegers, K. 2003. Software Requirements (2nd ed.). Redmond, WA: Microsoft Press. 